\title{
Mixed adenoneuroendocrine carcinoma of the liver: A rare case report
}

\author{
JIAO-JIAO YANG ${ }^{1}$, ZHI-PING LI ${ }^{1,2}$, CHUN-LI LUO ${ }^{1}$, YAN DU ${ }^{1}$, QIU-YANG LU ${ }^{1}$, \\ NA LI ${ }^{1}, \mathrm{HE} \mathrm{LI}^{1}$, TIAN-PING YU ${ }^{2}$ and XING-MING HUANG ${ }^{2}$ \\ ${ }^{1}$ Department of Radiotherapy in Cancer Center; ${ }^{2}$ Department of Pathology, \\ West China Hospital, Sichuan University, Chengdu, Sichuan 610041, P.R. China
}

Received February 25, 2019; Accepted August 16, 2019

DOI: $10.3892 / \mathrm{mco} .2019 .1962$

\begin{abstract}
A 55-year-old woman presented with chest and back pain of unknown cause. Contrast-enhanced computed tomography revealed two low-density tumors, sized 4.6 and $4.4 \mathrm{~cm}$, in the hepatic caudate and left inner lobes, respectively. There are multiple enlarged lymph nodes around the abdominal aorta, hepatogastric ligament and gastrosplenic ligament. At the same time, there were multiple enlarged lymph nodes between the portal vein and the vena cava. Upper gastrointestinal endoscopy revealed chronic non-atrophic gastritis and esophagitis (grade B). Endoscopic examination of the lower digestive tract revealed polyps of the colon, diagnosed as tubular adenomas following biopsy and histopathological examination. The patient underwent left three hepatic resection (including left inner lobe, left outer lobe and right anterior lobe resection), abdominal lymph node dissection, right liver tumor radiofrequency ablation, hepatic caudate lobe resection, intestinal adhesion release, vena cava formation, portal vein repair and hilar cholangioplasty. The pathological examination of the resected specimens revealed intrahepatic bile duct carcinoma and hepatic parenchymal neuroendocrine tumor (NET). In addition, liver solid portions consisted of tumor cells with characteristic salt-and-pepper nuclei. Immunohistochemical examination revealed expression of the neuroendocrine marker synaptophysin in this solid component, confirming the diagnosis of NET. Furthermore, the MIB-1 proliferation index of the NET was higher compared with that of the adenocarcinoma, and lymph node invasion by the NET component was detected, indicating a neuroendocrine carcinoma (NEC, or NET G3). The diagnosis of mixed adenoneuroendocrine carcinoma of the liver was confirmed based on the World Health Organization 2010 criteria. Taking into consideration the
\end{abstract}

Correspondence to: Professor Zhi-Ping Li, Department of Radiotherapy in Cancer Center, West China Hospital, Sichuan University, no 17, Section 3, Renmin South Road, Wuhou, Chengdu, Sichuan 610041, P.R. China

E-mail: lizhiping620312@163.com

Key words: mixed adenoneuroendocrine carcinoma, neuroendocrine carcinoma, adenocarcinoma, liver tumor patient's poor general condition, only symptomatic supportive treatment was administered postoperatively, without chemotherapy. Contrast-enhanced computed tomography at 45 days postoperatively revealed disease progression, with metastases in the liver stump, abdominal lymph nodes, spine and pelvis. The patient remained on symptomatic supportive treatment and succumbed to disease progression 3 months after surgery.

\section{Introduction}

The first gastrointestinal tumor with dual neuroendocrine and exocrine differentiation was reported by Cordier in 1924 (1). The World Health Organization published a new classification for neuroendocrine neoplasms (NENs) of the digestive system in 2010 that divided NENs into four main categories: Neuroendocrine tumor (NET) G1, NET G2, neuroendocrine carcinoma (NEC or NET G3), and mixed adenoneuroendocrine carcinoma (MANEC). MANEC is defined as a tumor that is composed of neuroendocrine and epithelial cells arranged in glandular formations, and may be considered as cancer, as both components are malignant. Occasionally, a squamous cell carcinoma component may be detected, albeit rarely. It is generally considered that these two components must account for at least $30 \%$ of the tumor in cases diagnosed with mixed cancer, as only a small number of immunohistochemically positive neuroendocrine cells found in adenocarcinoma are not sufficient to establish a diagnosis of mixed cancer (2).

Liver MANECs are relatively rare, with only a few cases reported to date, mainly in the stomach $(3,4)$, pancreas $(5)$, esophagus (6) and Vater ampulla (7). Due to the low incidence of hepatic MANECs, they may pose a diagnostic challenge, as only one component is usually identified and the diagnosis is incomplete. The diagnosis of MANECs mainly depends on pathological examination. Tumor architecture is the most important diagnostic characteristic of MANECs, and the diagnosis is then confirmed by immunohistochemical examination for chromogranin A, synaptophysin, CD56, or neuron-specific enolase (NSE) expression (8). The treatment of MANECs is mainly surgical, and prognosis depends on the stage and tumor type. There are currently no definitive recommendations regarding adjuvant chemotherapy due to the small number of such cases reported to date. Further improvements in the diagnosis and treatment of MANEC are needed. The purpose 
of the present study was to further explore the pathogenesis of MANEC, and to provide a theoretical basis for early diagnosis, clinical treatment and prognosis of this type of tumor, in order improve diagnostic awareness and optimize individualized treatment.

\section{Case report}

A 55-year-old woman presented with chest and back pain of unknown etiology. The patient was admitted to the West China Hospital (Chengdu, China) for further examination and treatment. Laboratory data revealed increased levels of $\alpha$-fetoprotein (AFP), serum carbohydrate antigen (CA)125 and NSE, which were $23.41 \mathrm{ng} / \mathrm{ml}$ (normal range $<20 \mathrm{ng} / \mathrm{ml}$ ), $62.26 \mathrm{U} / \mathrm{ml}$ and $127.9 \mathrm{ng} / \mathrm{ml}$, respectively. The AFP values associated with primary hepatocellular carcinoma are generally known to be $>500 \mathrm{ng} / \mathrm{ml}$ for 4 weeks, or $200-500 \mathrm{ng} / \mathrm{ml}$ for 8 weeks. Therefore, the diagnosis of primary liver cancer in this patient was not considered likely. CA125 is widely present in mesothelial tissue and is currently the most important ovarian cancer-associated antigen. CA125 is the most reliable diagnostic indicator for ovarian cancer, and its normal level is $<35 \mathrm{U} / \mathrm{ml}$, whereas NSE is mainly used for the diagnosis of neuroendocrine tumors, with a serum reference value of $<12.5 \mathrm{ng} / \mathrm{ml}$. Contrast-enhanced computed tomography (CT) examination revealed low-density tumors sized 4.6 and $4.4 \mathrm{~cm}$ in the caudate and left internal lobe of the liver, respectively (Fig. 1A and B). There were multiple enlarged lymph nodes along the abdominal aorta, the hepatogastric and gastrosplenic ligaments, and in the space between the portal vein and the inferior vena cava (Fig. 1C). The patient was subjected to left three hepatic resection (including left inner lobe, left outer lobe and right anterior lobe resection), abdominal lymph node dissection, liver tumor radiofrequency ablation, hepatic caudate lobe resection, intestinal adhesion release, repair of vena cava damage, portal vein repair and hilar cholangioplasty in our hospital. The liver margin of the surgical specimen was not invaded by cancer. Subsequent immunohistochemical examination did not rule out the possibility of a gastrointestinal origin, and gastrointestinal endoscopy and positron emission tomography (PET)-CT were performed to exclude distant metastasis. Upper gastrointestinal endoscopy revealed chronic non-atrophic gastritis and esophagitis (grade B). Endoscopic examination of the lower digestive tract detected polyps of the colon, diagnosed as tubular adenomas following biopsy and histopathological examination. The PET examination demonstrated active glucose metabolism in the liver, cervical lymph nodes, abdominal lymph nodes and bones, mostly due to tumor metastases (Fig. 2). There was no evidence of intestinal, ovarian or other metastases.

The surgical specimen was fixed in $10 \%$ neutral formalin solution for $>48 \mathrm{~h}$. The tissue samples were then embedded in paraffin for sectioning at $1 \mathrm{~mm}$, and the sections were subjected to conventional dewaxing and hydration. A multimeric anti-rabbit/mouse IgG-HRP kit (SV0004; Boster Biological Technology Co., Ltd.) was applied for experimental testing. The sections were incubated with $3 \% \mathrm{H}_{2} \mathrm{O}_{2}$ in deionized water for 5-10 $\mathrm{min}$ at room temperature to eliminate endogenous peroxidase activity, and then rinsed with PBS (Boster Biological Technology Co., Ltd.; cat. no. AR0030)
3 times for 5 min each time. The antigen was repaired by boiling $0.01 \mathrm{M}$ sodium citrate buffer solution in a microwave oven. After adding 5\% BSA blocking solution, the sections were incubated at $37^{\circ} \mathrm{C}$ for $30 \mathrm{~min}$ for blocking and then dried. The sections were then incubated with primary antibodies against CK20 (clone OVIL 12/30; Leica Biosystems; 1:50 dilution), carcinoembryonic antigen (CEA) (clone 12-140-10; Leica Biosystems; 1:200 dilution), synaptophysin (Merck KGaA; cat. no. MAB5258-I; 1:200 dilution), Ki-67 (BM2889; 1:100 dilution), retinoblastoma (RB; BM2184; 1:100 dilution) and p53 (BM0101; 1:50 dilution) (all from Boster Biological Technology Co., Ltd.) at $37^{\circ} \mathrm{C}$ for $1-2 \mathrm{~h}$, and then washed 3 times with PBS, 5 min each time. HRP-labeled anti-rabbit/mouse IgG was added dropwise, incubated at $37^{\circ} \mathrm{C}$ for $30 \mathrm{~min}$ and rinsed with PBS 3 times, $5 \mathrm{~min}$ each time. The sections were colored with DAB (the reaction time was controlled under a microscope) and then washed thoroughly with tap water. According to the need for hematoxylin (cat. no. AR0005; Boster Biological Technology Co., Ltd.) re-dyeing, the dyeing time was 0.5-2 $\mathrm{min}$. The sections were then dehydrated, transparentized and photographed under a microscope (Olympus CX31; Olympus Corporation).

The excised liver tumor was composed of two parts. One part was a tumor with nest-like growth in the liver parenchyma, and the nest-like areas consisted of highly malignant large cells (Fig. 3A). The nest-like areas lacking acinar/glandular structures consisted of tumor cells with salt-and-pepper nuclei, a high nucleus-to-cytoplasm ratio and increased nuclear chromatin density (Fig. 3D). The other part was an adenocarcinomatous component arising from the intrahepatic bile ducts (Fig. 3B). Under high magnification, the cells exhibited disorderly arrangement and prominent atypia, with scattered goblet cells and a large amount of mucus secreted in the lumen (Fig. 3C). The expression of cytokeratin (CK)20 and CEA, which are positive in intrahepatic cholangiocarcinoma, confirmed the adenocarcinomatous nature (Fig. 4A-D). Synaptophysin, which is positively expressed in hepatic parenchymal tumors and negative in bile duct adenocarcinoma, confirmed the NET component of MANEC and the dual differentiation of this tumor (Fig. 4E and F). The MIB-1 proliferation index of the NET component was significantly higher compared with that of the adenocarcinomatous component (Fig. 4G and $\mathrm{H}$ ). The Ki-67 positivity rate of the NET component was $\sim 60 \%$, and the mitotic figure count was $\sim 35 / 10$ high-power fields (HPF). Lymph node invasion by the NET component was identified (Fig. 3E), indicating that NET was the main determinant of the prognosis. RB and P53 are positive in NET and adenocarcinoma. RB and P53 positivity is observed in various tumors, and suggests a poor prognosis (Fig. 4I-L). The patient in this case succumbed to disease progression 3 months after surgery.

\section{Discussion}

According to the 2010 WHO classification, NETs of the digestive system are classified as follows: NET G1 (carcinoid; mitotic count: $<2$ per $10 \mathrm{HPF}$ and/or $\leq 2 \% \mathrm{Ki}-67$ index); NET G2 (mitotic count: $2-20$ per $10 \mathrm{HPF}$ and/or 3-20\% Ki-67 index); NEC (large- or small-cell type); and MANEC (9). The Ki-67 positivity rate in the present case was $\sim 60 \%$, and the mitotic 

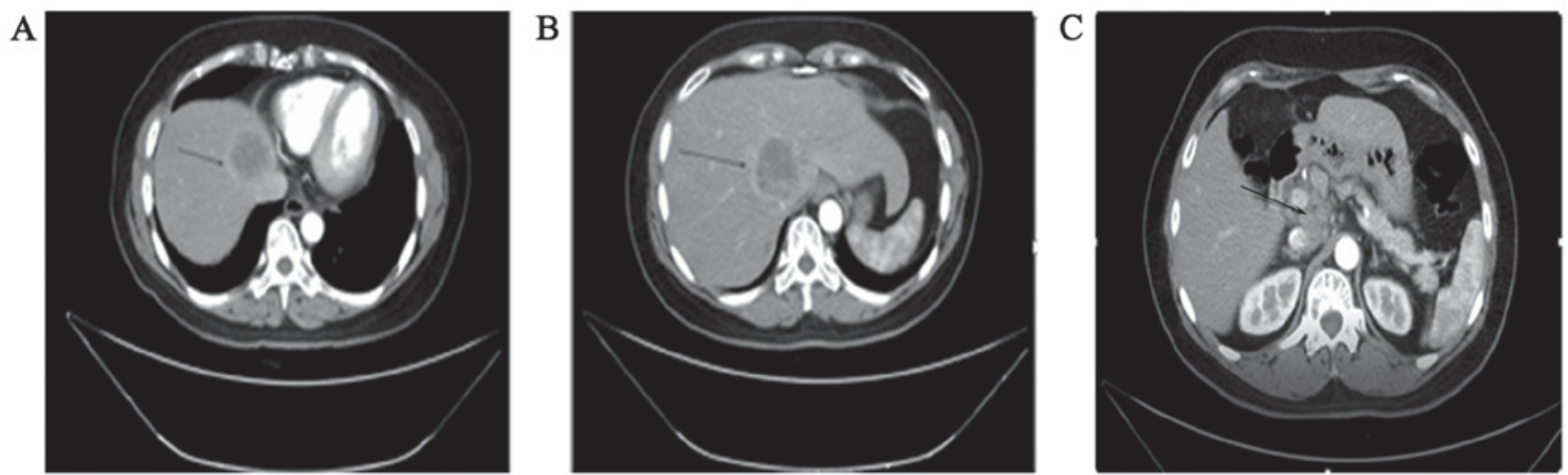

Figure 1. (A and B) Computed tomography of liver revealed two soft tissue masses of slightly lower density in the liver, sized $~ 4.6$ and $4.4 \mathrm{~cm}$, with enhancement of the margins (arrows). (C) Computed tomography of the abdomen revealed multiple lymph node enlargements along the abdominal aorta, hepatogastric and gastrosplenic ligaments and in the space between the portal vein and the inferior vena cava (arrow).
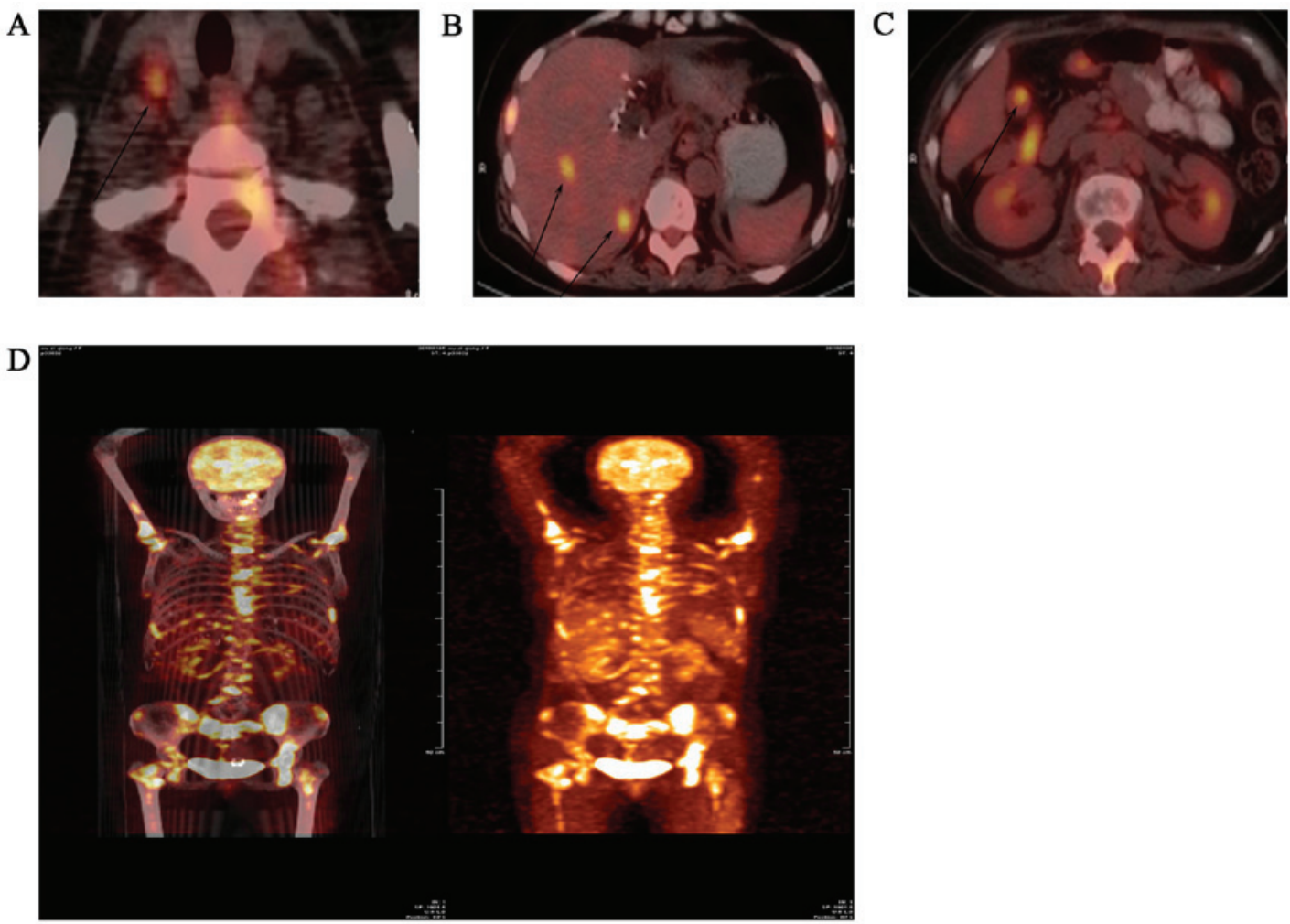

Figure 2. Positron emission tomography examination revealed increased glucose metabolism in (A) the neck lymph nodes, (B) abdominal lymph nodes and (C) the residual liver, whereas (D) multiple bone metastatic lesions were also detected.

figure count was $\sim 35 / 10$ HPF. The neuroendocrine components contain small, intermediate and large neuroendocrine cells.

In cytology, classification relies on differentiation (number of mitotic divisions and $\mathrm{Ki}-67$ proliferation index), which reflects the extent to which malignant cells resemble normal cells. Tumor morphology and Ki-67 proliferation index positivity rate are considered as key prognostic factors (10). According to the grade of differentiation and malignancy of the two components, MANECs are further divided into the following subtypes: High-grade malignant MANEC, intermediate-grade malignant MANEC, and a low-grade mixed gonadal neuroendocrine tumor (11). High-grade malignant MANECs consist of a carcinomatous component (adenocarcinoma or squamous cell carcinoma) and a poorly differentiated NEC component. Intermediate-grade malignant MANECs consist of adenocarcinoma and G1 or G2 NEN, whereas low-grade mixed gonadal neuroendocrine tumors consist of adenoma and G1 or G2 NEN components. Based on the Digestive System Tumor NET WHO 2010 Grading Criteria, NET G3 (NEC) is defined as a mitotic count $>20 / 10 \mathrm{HPF}$ and/or Ki-67 >20\%. Therefore, the NET of the patient in the present case was G3 (NEC).

CK20 and CEA are positive in adenocarcinoma, and confirmed the presence of an adenocarcinomatous component in MANEC. Synaptophysin, which is positive in NET and negative in adenocarcinoma, confirmed the NET component of the MANEC. RB and P53 expression is positive in various tumors, and is suggestive of a poor prognosis. It was previously 
A

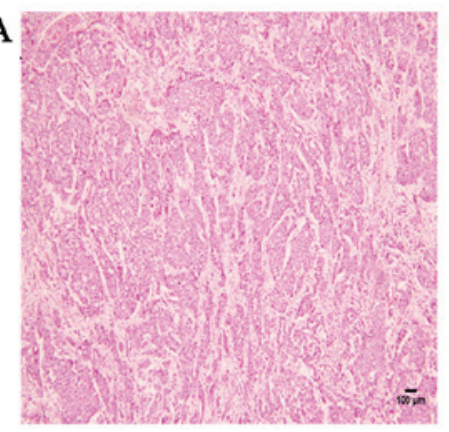

D

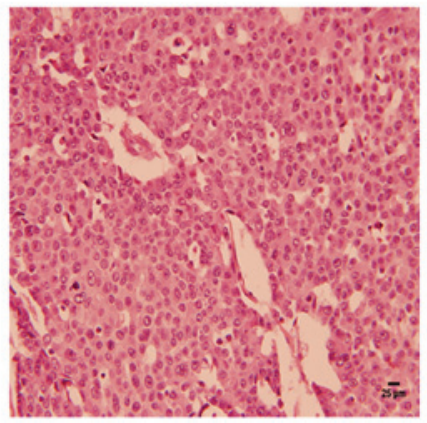

B
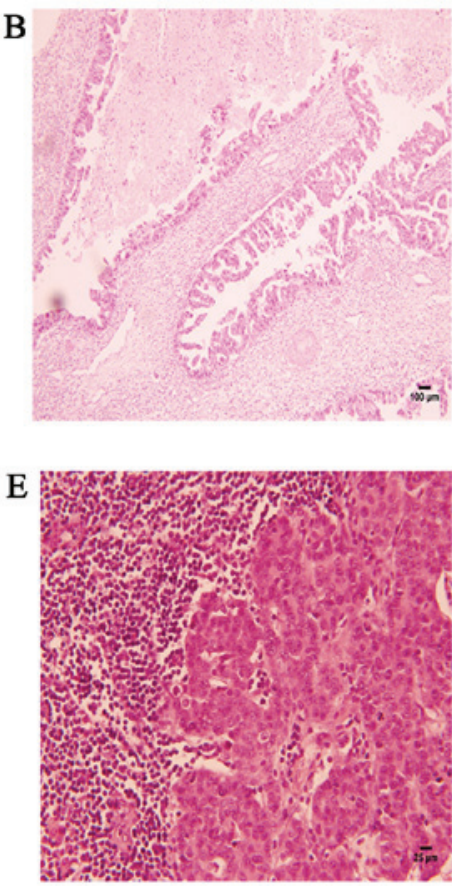

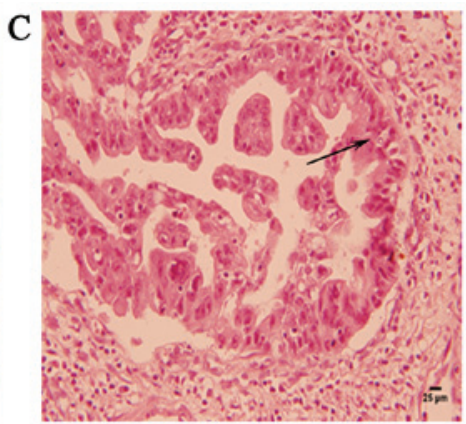

Figure 3. Pathological findings from the resected liver (hematoxylin eosin staining). (A) Tumor cell nests found in the parenchyma of the resected liver, composed of highly malignant large cells; magnification, x100. (B) Adenocarcinoma component originating from the intrahepatic bile duct; magnification, x100. (C) Under high magnification (x400), adenocarcinoma cells exhibited disorderly arrangement and high atypia, with scattered goblet cells (arrow) and a large amount of mucus secreted in the lumen. (D) The nest-like areas lacked acinar/glandular structures and comprised tumor cells with salt-and-pepper nuclei, a high nucleus-to-cytoplasm ratio, and increased nuclear density; magnification, $\mathrm{x} 400$. (E) Lymph node invasion by the neuroendocrine component was identified; magnification, x400; H\&E staining

suggested that, when MANECs contain poorly differentiated (G3) NET components, they should be treated as NECs $(12,13)$. This simpler proliferation-based ENETS/WHO 2010 classification system provides prognostic information, but has not been validated to predict recurrence following surgical resection.

Liver MANEC is rarely reported. The origin of MANEC remains uncertain. There are two main theories regarding the origin of this disease. Based on the first theory, it is hypothesized that the two components of MANEC originate from two different cell lines. The adenocarcinoma cells originate from pluripotent stem cells, whereas NEC originates from embryonic neural cells. Based on the second hypothesis, MANEC is considered to originate from endodermal pluripotent stem cells, which are affected by hormones, the local microenvironment and an instable genome during the process of tumor occurrence and development, eventually leading to a two-way or multidirectional differentiation (14-16). However, in most cases, the adenocarcinomatous and NEC components of MANEC are cross-mixed. Only in a few cases these two components are closely linked without mixing (referred to as 'colliding' tumors), which suggests that the majority of these tumors may originate from pluripotent stem cells and undergo multidirectional differentiation during tumor occurrence and development. Zhang et al (17) reported that, in addition to the adenocarcinoma and NEC components, mixed cancer also contains squamous cell carcinoma, which may support the concept that these tumors originate from pluripotent stem cells. However, it remains unclear why two tumor cells of a different origin and behavior can coexist in one tumor, that is, that the same tumor contains two different components of cancer cells, adenocarcinoma or neuroendocrine cancer and the squamous cell carcinoma.

It was previously considered that the percentage of each component in mixed cancers determines disease progression. However, retrospective case analyses have demonstrated that the prognosis of MANEC depends on the main component (18), rather than the proportion of each component. This is because this smaller percentage volume may metastasize and significantly affect patient outcome. Most authors argue that the characteristics of the neuroendocrine component have a considerable impact on the clinical manifestations of MANEC (19). In addition, in most literature reports, lymph node and liver metastases are almost always from NEC rather than adenocarcinoma, as NECs are usually poorly differentiated and more aggressive compared with adenocarcinomas. Scardoni et al (20) concluded that there are multiple drivers of gene mutations in the neuroendocrine component, such as the ATM, ERBB4, KDR/VEGFR2, JAK3 and TP53 genes. Although some authors believe that clinical behavior depends on the grade of the neuroendocrine component, others report that the characteristics of the adenocarcinomatous part affect the outcome in cases with well-differentiated neuroendocrine components $(21,22)$. Gurzu et al $(8)$ reported that the glandular component was predominant in lymph node metastases, and nuclear expression of maspin in glandular structures compared with its negativity in the neuroendocrine component confirmed the higher aggressiveness of adenocarcinoma compared with poorly differentiated NEC. A previous study reported that the mortality rate by histological type was significantly higher in low differentiation adenocarcinoma/adverse NET compared with that in highly 
A

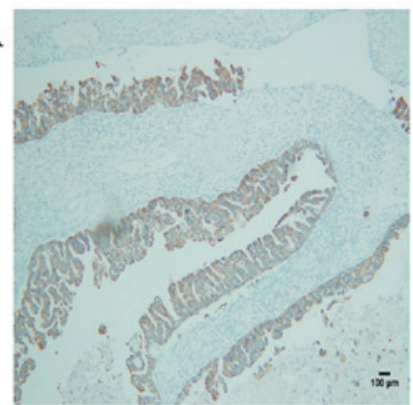

B

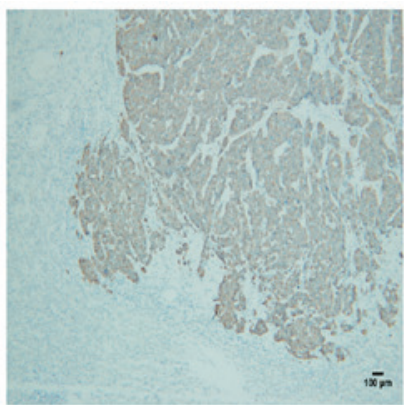

D

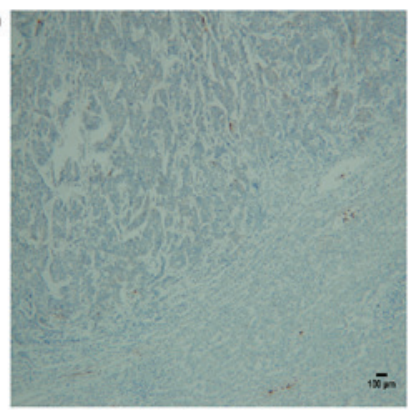

G
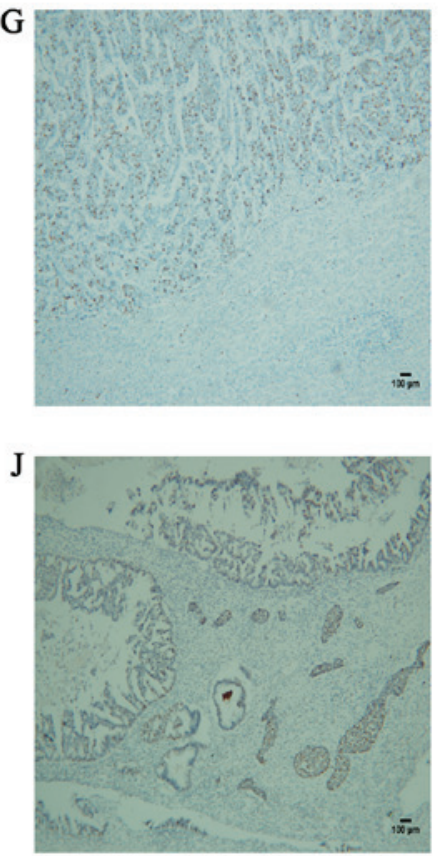

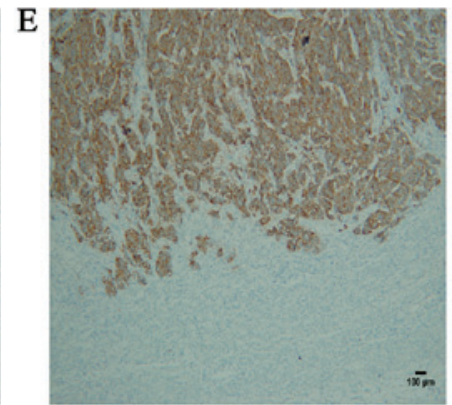

$\mathrm{H}$
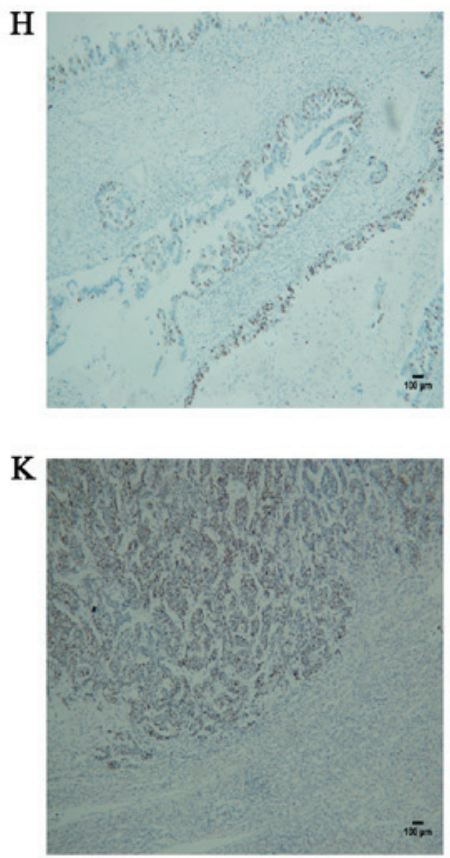
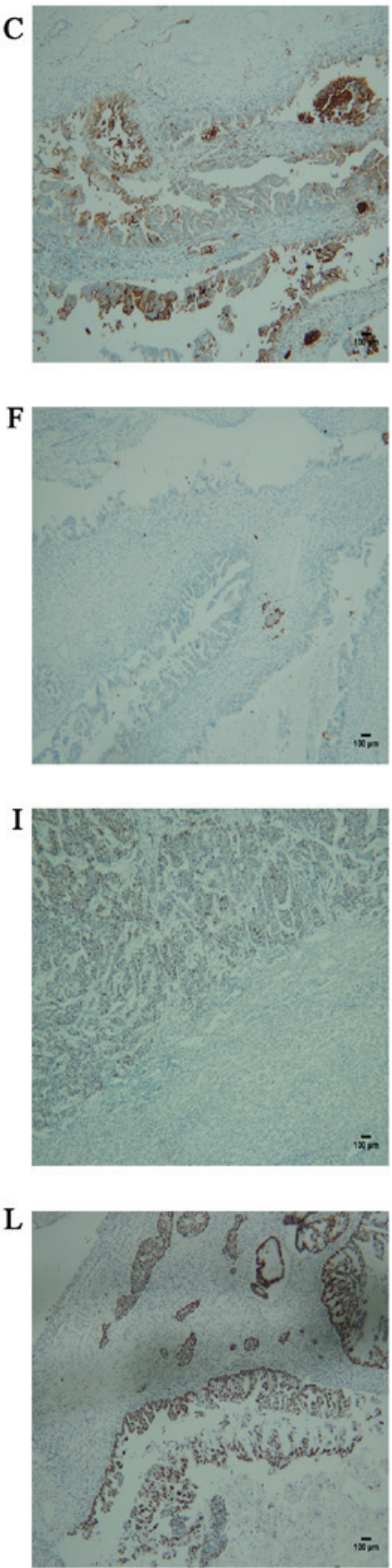

Figure 4. Immunohistochemical staining. CK20 staining was positive in (A) adenocarcinoma and (B) NET; magnification, x100. Carcinoembryonic antigen staining was (C) positive in adenocarcinoma and (D) negative in NET; magnification, x100. Synaptophysin staining was (E) positive in NET and (F) negative in adenocarcinoma; magnification, x100. The MIB-1 proliferation index of (G) NET was higher compared with that of (H) adenocarcinoma; magnification, x100. RB staining was positive in (I) NET and (J) adenocarcinoma; magnification, x100. P53 staining was positive in (K) NET and (L) adenocarcinoma; magnification, x100. CK, cytokeratin; NET, neuroendocrine tumor; RB, retinoblastoma gene.

differentiated (adenocarcinoma/low-grade NETs (23). This suggests that the NEC component, and not the adenocarcinoma component, is the main driver of cancer progression in this cancer type. Others believe that adenocarcinoma may affect the prognosis of MANECs with highly differentiated NEC. However, in the present case, both the adenocarcinoma and NEC were high-grade malignant tumors (the NEC component accounted for $\sim 60 \%$ and the adenocarcinoma for $\sim 40 \%$ ). The metastatic lymph nodes in this case were from NEC, which suggests that NEC is more likely to invade lymphatic vessels and plays an important role in the prognosis of high-grade
MANEC, which is mainly responsible for disease progression. Based on most literature reports, lymph node metastasis is almost always from NEC rather than adenocarcinoma and, therefore, must be treated as a NEC. In addition, the higher the positive rate of P53 expression on immunohistochemistry, the worse the prognosis. The RB gene is widely distributed in various tissues, and is known to inhibit cell proliferation, promote cell differentiation, and regulate the cell cycle, but its role in MANEC remains unclear, although it may be associated with prognosis. Further research on the RB gene is required. $\mathrm{Ki}-67$ is a marker of cell proliferation and, the higher its 
positivity rate, the higher the malignant potential of the tumor. $\mathrm{Ki}-67$ is closely associated with tumor differentiation, invasion, metastasis and prognosis. However, further extensive clinical research and epidemiological analysis are required. A clear understanding of the key factors affecting cancer progression is crucial for determining standard treatment options. However, recent WHO classifications indicate that MANEC treatment is similar to that for common adenocarcinoma.

Due to the rarity of MANEC, the most effective chemotherapy regimen remains to be determined. The National Comprehensive Cancer Network recommends the use of cisplatin or etoposide, but the prognosis remains poor, with a median survival time of 7-10 months at present. However, there is currently no consensus among different investigators regarding the choice of adjuvant chemotherapy. Some authors recommend the use of cisplatin or carboplatin with etoposide (24), whereas others approve the combination of cisplatin with fluoropyrimidines in cases with metastatic adenopathy (25). Surgery remains the preferred treatment for early liver malignant tumors. For the diagnosis of liver malignant tumors, a bioptic specimen may be collected during surgery. However, a liver biopsy may lead to dissemination of tumor cells, which may cause metastasis to the peritoneum or other sites and worsen the prognosis. Based on each patient's status, the development of effective and individualized treatment approaches to reduce postoperative complications requires continuous clinical practice and experience.

\section{Acknowledgements}

Not applicable.

\section{Funding}

No funding was received.

\section{Availability of data and materials}

Datasets used and/or analyzed during the present study are available from the corresponding author on reasonable request.

\section{Authors' contributions}

JJY, ZPL, TPY and XMH participated, conceived and designed the present case report, analyzed and interpreted the data and wrote the manuscript. ZPL, CLL and YD evaluated the patient and participated in treatment. YD and CLL evaluated radiological images. QYL made strict changes to the language of the manuscript and made suggestions. NL and HL participated in the histochemical photography and scrutinized the manuscript. All authors have read and approved the final version of the manuscript.

\section{Ethics approval and consent to participate}

Not applicable.

\section{Patient consent for publication}

The patient's family provided permission to publish the case details and any accompanying images.

\section{Competing interests}

The authors declare that they have no competing interests.

\section{References}

1. Jain A, Singla S, Jagdeesh KS and Vishnumurthy HY: Mixed adenoneuroendocrine carcinoma of cecum: A rare entity. J Clin Imaging Sci 3: 10, 2013.

2. Shimada N, Miwa S, Arai T, Kitagawa N, Akita S, Iinuma N and Ishii K: Cystic mixed adenoneuroendocrine carcinoma of the pancreas: A case report. Int J Surg Case Rep 52: 1-4, 2018.

3. Kwok CM: Mixed adenoneuroendocrine carcinoma of the stomach. Case Rep Gastroenterol 9: 241-245, 2015.

4. Yamauchi H, Sakurai S, Nakazawa N, Yoshida T, Tabe Y, Saitoh K, Fukasawa T, Kiriyama S, Naitoh H and Kuwano H: A case of mixed adenoneuroendocrine carcinoma of the stomach with focal intestinal metaplasia and hypergastrinemia. Int Surg 100: 562-567, 2015.

5. Minakawa K, Oka K, Nihei T, Sando N, Oikawa H, Toda J, Hosokawa Y, Matsumoto T and Yanagisawa A: Pancreatic endocrine tumor with partial acinar cell differentiation. APMIS 114: 720-725, 2006

6. Kadhim MM, Jespersen ML, Pilegaard HK, Nordsmark M and Villadsen GE: Mixed adenoneuroendocrine carcinoma is a rare but important tumour found in the oesophagus. Case Rep Gastrointest Med 2016: 9542687, 2016.

7. Ginori A, Lo Bello G, Vassallo L and Tripodi SA: Amphicrine carcinoma of the ampullary region. Tumori 101: e70-e72, 2015.

8. Gurzu S, Kadar Z, Bara T, Bara T Jr, Tamasi A, Azamfirei L and Jung I: Mixed adenoneuroendocrine carcinoma of gastrointestinal tract: Report of two cases. World J Gastroenterol 21: 1329-1333, 2015.

9. Onishi I, Kitagawa H, Harada K, Maruzen S, Sakai S, Makino I, Hayashi $\mathrm{H}$, Nakagawara $\mathrm{H}$, Tajima $\mathrm{H}$, Takamura $\mathrm{H}$, et al: Intraductal papillary neoplasm of the bile duct accompanying biliary mixed adenoneuroendocrine carcinoma. World J Gastroenterol 19: 3161-3164, 2013.

10. Niederle MB, Hackl M, Kaserer $K$ and Niederle B: Gastroenteropancreatic neuroendocrine tumours: The current incidence and staging based on the WHO and European neuroendocrine tumour society classification: An analysis based on prospectively collected parameters. Endocr Relat Cancer 17: 909-918, 2010.

11. Tang Q, Zhou Z, Chen J, Di M, Ji J, Yuan W, Liu Z, Wu L, Zhang X, Li K and Shu X: Correlation of metastasis characteristics with prognosis in gastric mixed adenoneuroendocrine carcinoma: Two case reports. Medicine (Baltimore) 96: e9189, 2017.

12. La Rosa S, Marando A, Sessa F and Capella C: Mixed Adenoneuroendocrine carcinomas (MANECs) of the gastrointestinal tract: An update. Cancers (Basel) 4: 11-30, 2012.

13. Volante M, Rindi G and Papotti M: The grey zone between pure (neuro)endocrine and non-(neuro)endocrine tumours: A comment on concepts and classification of mixed exocrineendocrine neoplasms. Virchows Arch 449: 499-506, 2006.

14. Furlan D, Cerutti R, Genasetti A, Pelosi G, Uccella S, La Rosa S and Capella C: Microallelotyping defines the monoclonal or the polyclonal origin of mixed and collision endocrine-exocrine tumors of the gut. Lab Invest 83: 963-971, 2003.

15. Paniz Mondolfi AE, Slova D, Fan W, Attiyeh FF, Afthinos J, Reidy J, Pang Y and Theise ND: Mixed adenoneuroendocrine carcinoma (MANEC) of the gallbladder: A possible stem cell tumor? Pathol Int 61: 608-614, 2011.

16. Vanacker L, Smeets D, Hoorens A, Teugels E, Algaba R, Dehou MF, De Becker A, Lambrechts D and De Greve J: Mixed adenoneuroendocrine carcinoma of the colon: Molecular pathogenesis and treatment. Anticancer Res 34: 5517-5521, 2014.

17. Zhang W, Xiao W, Ma H, Sun M, Chen H and Zheng S: Neuroendocrine liver metastasis in gastric mixed adenoneuroendocrine carcinoma with trilineage cell differentiation: A case report. Int J Clin Exp Pathol 7: 6333-6338, 2014.

18. Lee EJ, Park SM, Maeng L, Lee A and Kim KM: Composite glandular-endocrine cell carcinomas of the stomach: Clinicopathologic and methylation study. APMIS 113: 569-576, 2005. 
19. Levi Sandri GB, Carboni F, Valle M, Visca P and Garofalo A: Mixed adenoneuroendocrine gastric carcinoma: A case report and review of the literature. J Gastric Cancer 14: 63-66, 2014.

20. Scardoni M, Vittoria E, Volante M, Rusev B, Bersani S, Mafficini A, Gottardi M, Giandomenico V, Malleo G, Butturini G, et al: Mixed adenoneuroendocrine carcinomas of the gastrointestinal tract: Targeted next-generation sequencing suggests a monoclonal origin of the two components. Neuroendocrinology 100: 310-316, 2014.

21. Kim JJ, Kim JY, Hur H, Cho YK and Han SU: Clinicopathologic significance of gastric adenocarcinoma with neuroendocrine features. J Gastric Cancer 11: 195-199, 2011.

22. Lee JH, Kim HW, Kang DH, Choi CW, Park SB and Kim SH: A gastric composite tumor with an adenocarcinoma and a neuroendocrine carcinoma: A case report. Clin Endosc 46: 280-283, 2013.

23. Li Y, Yau A, Schaeffer D, Magliocco A, Gui X, Urbanski S, Waghray R, Owen D and Gao ZH: Colorectal glandular-neuroendocrine mixed tumor: Pathologic spectrum and clinical implications. Am J Surg Pathol 35: 413-425, 2011.
24. Delle Fave G, Kwekkeboom DJ, Van Cutsem E, Rindi G, Kos-Kudla B, Knigge U, Sasano H, Tomassetti P, Salazar R and Ruszniewski P: ENETS Consensus Guidelines for the management of patients with gastroduodenal neoplasms. Neuroendocrinology 95: 74-87, 2012.

25. Van Laethem JL, Carneiro F, Ducreux M, Messman H, Lordick F, Ilson DH, Allum WH, Haustermans K, Lepage C, Matysiak-Budnik T, et al: The multidisciplinary management of gastro-oesophageal junction tumours: European society of digestive oncology (ESDO): Expert discussion and report from the 16th ESMO world congress on gastrointestinal cancer, barcelona. Dig Liver Dis 48: 1283-1289, 2016.

(i) This work is licensed under a Creative Common Attribution-NonCommercial-NoDerivatives 4.0 International (CC BY-NC-ND 4.0) License. 